Check for updates

Department of Epidemiology Research, Statens Serum Institut, Copenhagen, Denmark

Correspondence to: $\mathrm{H}$ Boyd hoy@ssi.dk

(ORCID 0000-0001-6849-9985)

Additional material is published online only. To view please visit the journal online.

Cite this as: BMJ 2018;363:k4109 http://dx.doi.org/10.1136/bmi.k4109

Accepted: 15 September 2018

\title{
Pre-eclampsia and risk of dementia later in life: nationwide cohort study
}

\author{
Saima Basit, Jan Wohlfahrt, Heather A Boyd
}

\section{ABSTRACT}

OBJECTIVE

To explore associations between pre-eclampsia and later dementia, overall and by dementia subtype and timing of onset.

DESIGN

Nationwide register based cohort study.

SETTING

Denmark.

\section{POPULATION}

All women with at least one live birth or stillbirth between 1978 and 2015.

\section{MAIN OUTCOME MEASURE}

Hazard ratios comparing dementia rates among women with and without a history of pre-eclampsia, estimated using Cox regression.

\section{RESULTS}

The cohort consisted of 1178005 women with 20352695 person years of follow-up. Women with a history of pre-eclampsia had more than three times the risk of vascular dementia (hazard ratio $3.46,95 \%$ confidence interval 1.97 to 6.10 ) later in life, compared with women with no history of preeclampsia. The association with vascular dementia seemed to be stronger for late onset disease (hazard ratio $6.53,2.82$ to 15.1 ) than for early onset disease $(2.32,1.06$ to 5.06$)(P=0.08)$. Adjustment for diabetes, hypertension, and cardiovascular disease attenuated the hazard ratios only moderately; sensitivity analyses suggested that body mass index

\section{WHAT IS ALREADY KNOWN ON THIS TOPIC}

Women with a history of hypertensive disorders of pregnancy show signs of cognitive impairment and brain atrophy after an affected pregnancy, both in the short term and decades later

The STOX1 gene, a variant of which increases susceptibility to pre-eclampsia, is overexpressed in late onset Alzheimer's disease

Epidemiological studies have found limited evidence of a direct association between hypertensive disorders of pregnancy and dementia, but these studies had limitations

\section{WHAT THIS STUDY ADDS}

Women with a history of pre-eclampsia had more than three times the risk of vascular dementia later in life compared with women with no history of preeclampsia

The association with vascular dementia seemed to be stronger for late onset disease (age $\geq 65$ years) than for early onset disease (age $<65$ years)

Adjustment for diabetes, hypertension, and cardiovascular disease attenuated the hazard ratios only moderately

Pre-eclampsia was only modestly associated with the risk of Alzheimer's disease, and the association may be accounted for by uncontrolled confounding by obesity was unlikely to explain the association with vascular dementia. In contrast, only modest associations were observed for Alzheimer's disease (hazard ratio 1.45, 1.05 to 1.99$)$ and other/unspecified dementia (1.40, 1.08 to 1.83$)$.

\section{CONCLUSIONS}

Pre-eclampsia was associated with an increased risk of dementia, particularly vascular dementia. Cardiovascular disease, hypertension, and diabetes were unlikely to mediate the associations substantially, suggesting that pre-eclampsia and vascular dementia may share underlying mechanisms or susceptibility pathways. Asking about a history of pre-eclampsia could help physicians to identify women who might benefit from screening for early signs of disease, allowing for early clinical intervention.

\section{Introduction}

Pre-eclampsia is characterised by new onset of hypertension and other signs of organ dysfunction arising during pregnancy. ${ }^{1}$ Endothelial and vascular dysfunction play a notable role in the pathophysiology of this disorder, ${ }^{23}$ and the vascular consequences of pre-eclampsia seem not to be confined to pregnancy: women with a history of pre-eclampsia have increased risks of later hypertension, cardiovascular disease, stroke, and diabetes. ${ }^{4-7}$ Given its vascular underpinnings, pre-eclampsia might therefore logically also be associated with an increased risk of dementia; recent research findings offer indirect support for this hypothesis. Pre-eclampsia has been associated with self reported impairment of cognitive function and white matter lesions confirmed by magnetic resonance imaging within a decade of an affected pregnancy, ${ }^{8} 9$ as well as with impaired cognition and brain atrophy decades later. $^{10}$ Moreover, the pre-eclampsia susceptibility gene STOX1 is overexpressed in the brain in late onset Alzheimer's disease. ${ }^{11}$ However, two cohort studies found limited evidence of an association between hypertensive disorders of pregnancy and later dementia, ${ }^{12} 13$ and a case-control study found that hypertensive disorders of pregnancy might be associated with early but not late onset Alzheimer's disease. ${ }^{14}$ On the other hand, all three studies were potentially underpowered to detect associations with an outcome occurring many decades after pregnancy. Furthermore, two of the studies were limited by their use of self reported information on complications of pregnancy and the attendant possibility of both recall bias and survival bias.

To overcome the limitations of the previous studies, we did a nationwide register based cohort study in more than 1.1 million women with pregnancies in 
Denmark in 1978-2015 and compared the risks of dementia between women with and without a history of pre-eclampsia, overall and by subtype and timing of onset of dementia.

\section{Methods}

Data sources

The Danish Civil Registration System registers all Danish residents by using unique personal identification numbers and updates information on demographics and vital status daily. ${ }^{15}$ The Medical Birth Register contains information on all live births and stillbirths in Denmark since 1973, with gestational age at delivery recorded since $1978 .{ }^{16}$ The National Patient Register contains information on all hospital discharge diagnoses assigned since 1977 and all outpatient diagnoses assigned since 1995, registered using ICD (international classification of diseases) codes. ${ }^{17}$ The Causes of Death Register has recorded the causes (underlying and contributing) of all deaths in Denmark since $1970 .^{18}$

\section{Cohort}

Using the Civil Registration System, we constructed a cohort of all women in Denmark with at least one pregnancy lasting 20 weeks or longer and ending in live birth or stillbirth between 1978 and 2015 (fig 1). Women were followed from their first delivery or 1 January 1994 (when ICD-10 codes were introduced in Denmark, allowing registration of dementia subtypes), whichever came later, until the first of dementia, death, emigration, designated "missing" in the Civil Registration System, or 31 May 2017 (end of followup). Women who died or emigrated before 1994 were excluded from the cohort, as were women with a diagnosis of ischaemic heart disease, myocardial infarction, stroke, heart failure, or diabetes registered in the National Patient Register before their first birth in the period 1978-2015. Women with a diagnosis of dementia before the start of follow-up were also excluded from the cohort.

\section{Pre-eclampsia (exposure)}

We considered a woman to have pre-eclampsia in a given pregnancy if she was registered with preeclampsia, eclampsia, or the HELLP (haemolysis, elevated liver enzymes, and low platelets) syndrome in the National Patient Register (ICD-8 codes

\section{i1 194381}

Women with at least one pregnancy lasting $\geq 20$ weeks in Denmark in 1978-2015

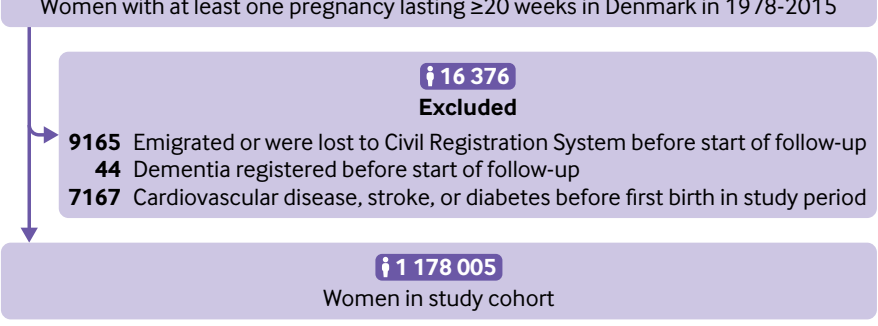

Fig 1 | Study cohort assembly and exclusions
637.03, 637.04, 637.09, 637.19, 637.99, 762.19, 762.29, 762.39; ICD-10 codes 014.0-015.9) after 20 weeks' gestation. We considered it unlikely that women with pre-eclampsia, the more severe forms in particular, would be registered with a diagnosis of pre-eclampsia early in pregnancy without also having confirmatory diagnoses registered around the time of delivery. Consequently, to be conservative and to improve the probability that women registered as having pre-eclampsia truly had pre-eclampsia, we also required that at least one diagnosis of preeclampsia be registered in the period from one month before delivery to seven days postpartum; we did not consider women whose only pre-eclampsia diagnoses were registered outside this period to have had pre-eclampsia. We treated history of pre-eclampsia as a time dependent variable, such that a woman could contribute person time in more than one exposure category during followup. However, once a woman had pre-eclampsia, she could not revert to the unexposed category; we assigned all subsequent person time to the "history of pre-eclampsia" category, even if she later had normotensive pregnancies.

In sub-analyses, we further categorised preeclampsia on the basis of gestational age at delivery, a proxy for timing of onset and severity of pre-eclampsia. We defined preterm pre-eclampsia as pre-eclampsia with delivery before 37 weeks' gestation and term preeclampsia as pre-eclampsia with delivery at or after 37 weeks' gestation. Also considered a time dependent history variable, at any given time we classified a women according to the earliest onset of pre-eclampsia she had had to date (if any).

\section{Dementia (outcome)}

We defined dementia as registration of any dementia code (ICD-10 codes F00.0-F02.0, F03.9, G30.0, G30.1, G30.8, G30.9) in the National Patient Register during follow-up. We further classified dementia as Alzheimer's disease (ICD-10 codes F00.0-F00.9, G30.0, G30.1, G30.8, G30.9), vascular dementia (ICD-10 codes F01.0-F01.9), and other/unspecified dementia (ICD-10 codes F02.0, F03.9). (We also used the ICD-8 codes 290.00, 290.10, 290.11, 290.19, and 299.99 to exclude women diagnosed as having dementia before the start of follow-up.)

\section{Covariates}

We considered parity (number of live births and/or stillbirths: one, two, and three or more), maternal birth year (five year intervals), region in which the child was delivered (a surrogate for socioeconomic status; table 1) and maternal age as a priori confounders. We treated all but birth year as time dependent variables.

We also investigated the influence of development of cardiovascular disease, stroke, chronic kidney disease, diabetes, and hypertension during follow-up. We identified women who developed these conditions during the follow-up period by using the National 


\begin{tabular}{|c|c|c|}
\hline \multirow[b]{2}{*}{ Characteristics at start of follow-up } & \multicolumn{2}{|c|}{ History of pre-eclampsia at start of follow-upt } \\
\hline & Yes $(n=50068 ; 4.3 \%)$ & No $(n=1127937 ; 95.7 \%)$ \\
\hline \multicolumn{3}{|l|}{ Age (years): } \\
\hline$<25$ & $6513(3.9)$ & $160299(96.1)$ \\
\hline $25-29$ & $14948(4.2)$ & $341268(95.8)$ \\
\hline $30-34$ & $13707(4.5)$ & $288127(95.5)$ \\
\hline $35-39$ & $8584(4.5)$ & $181479(95.5)$ \\
\hline $40-44$ & $4153(3.8)$ & $104253(96.2)$ \\
\hline $45-49$ & $1674(3.8)$ & $42900(96.2)$ \\
\hline$\geq 50$ & $489(4.8)$ & $9611(95.2)$ \\
\hline \multicolumn{3}{|l|}{ Maternal birth year: } \\
\hline$\leq 1944$ & $681(4.6)$ & $14155(95.4)$ \\
\hline $1945-49$ & $1912(3.6)$ & $51669(96.4)$ \\
\hline $1950-54$ & $4235(3.8)$ & $107375(96.2)$ \\
\hline $1955-59$ & $6719(4.6)$ & $140695(95.4)$ \\
\hline $1960-64$ & $8006(4.8)$ & $158207(95.2)$ \\
\hline $1965-69$ & $7552(4.3)$ & $167093(95.7)$ \\
\hline $1970-74$ & $7028(4.2)$ & $160195(95.8)$ \\
\hline $1975-79$ & $5921(4.0)$ & $141970(96.0)$ \\
\hline$\geq 1980$ & $8014(4.1)$ & $186578(95.9)$ \\
\hline \multicolumn{3}{|l|}{ Parity: } \\
\hline 1 & $33749(4.1)$ & $783784(95.9)$ \\
\hline 2 & $11558(4.3)$ & $255438(95.7)$ \\
\hline$\geq 3$ & $4761(5.1)$ & $88715(94.9)$ \\
\hline \multicolumn{3}{|l|}{ Region of latest delivery‡: } \\
\hline Greater Copenhagen & $14613(3.9)$ & $364794(96.1)$ \\
\hline Zealand§ & $5549(4.4)$ & $120681(95.6)$ \\
\hline Lolland, Falster, and Møn & $1261(4.6)$ & $26360(95.4)$ \\
\hline Bornholm and other & $307(2.3)$ & $13151(97.7)$ \\
\hline Funen and southern islands & $5495(5.6)$ & $93265(94.4)$ \\
\hline Southern Jutland & $6689(4.5)$ & $141469(95.5)$ \\
\hline Mid-Jutland & $10762(4.1)$ & $253138(95.9)$ \\
\hline Northern Jutland & $5392(4.5)$ & $115079(95.5)$ \\
\hline \multicolumn{3}{|c|}{$\begin{array}{l}\text { *Follow-up began on } 1 \text { January } 1994 \text { for women with births before } 1994 \text { and on date of first delivery for women } \\
\text { whose first birth occurred in or after } 1994 \text {. } \\
\text { †Totals reflect number of women with pre-eclampsia in pregnancies occurring before start of follow-up. Because } \\
\text { pre-eclampsia was time dependent variable, additional women developed pre-eclampsia during follow-up. } \\
\text { ‡Region of woman’s last delivery before start of follow-up. } \\
\text { §Areas of Zealand outside greater Copenhagen metropolitan area. }\end{array}$} \\
\hline
\end{tabular}

Patient Register and the Causes of Death Register, based on the following ICD-10 codes: myocardial infarction, I21.0-I23.9; ischaemic heart disease, I20.0-I20.9, I24.0-I24.9, or I25.0-I25.9; heart failure, I50.0-I50.9; stroke, I63.0-I63.9; chronic kidney disease, I12.0-I13.9, N18.0-N18.9 (and ICD-8 codes 400.39, 403.99, 404.99); diabetes, E10.0-E14.9. (We also used the following ICD-8 codes to exclude women diagnosed as having cardiovascular disease or diabetes before their first delivery: myocardial infarction, 410. $\mathrm{xx}$; ischaemic heart disease, 411.xx-414.xx, 420.xx429.xx; stroke, 433.xx, 436.xx; heart failure, 427.09427.19, 427.99, 428.99, 782.49; diabetes, 249.00250.09). We identified post-pregnancy hypertension on the basis of the filling of two prescriptions for antihypertensive drugs (Anatomic Therapeutic Chemical codes C02-03, C07-09 registered in the National Prescription Register) within a six month period. When defining hypertension, we ignored antihypertensive drug use that was potentially related to treatment of hypertensive disorders of pregnancy (use from 20 weeks before delivery to three months postpartum). We treated all comorbidities as time dependent variables.

\section{Statistical analysis}

We used Cox regression with age as the underlying time to estimate hazard ratios for dementia comparing women with and without a history of pre-eclampsia. We used competing risk methods when analysing associations with dementia subtypes. ${ }^{19}$ We checked the proportional hazards assumption by plotting cumulative Martingale residuals against age. ${ }^{20}$ We evaluated potential mediation by cardiovascular disease, stroke, chronic kidney disease, diabetes, and hypertension in an approximate way by adjusting for these variables. We used SAS statistical software, version 9.4 for all analyses.

\section{Sensitivity analyses}

We could not exclude women with pre-pregnancy hypertension from our main analyses, because registration of hypertension diagnoses in the National Patient Register is incomplete and information on antihypertensive drug use first became available in 1994. However, we did sensitivity analyses in which we excluded from the cohort women using antihypertensive drugs in 1994 (the year registration of filled prescriptions began) or before their first pregnancy, if this occurred in 1995 or later. Follow-up for these analyses began in 1995.

As pre-eclampsia accompanied by fetal growth restriction can be a sign of a particularly severe vascular phenotype, we did additional analyses stratified according to whether or not a pregnancy resulted in delivery of a small for gestational age infant (a proxy for fetal growth restriction). We defined small for gestational age as infant birth weight under the 10th centile for all babies of the same sex and birth cohort (five year intervals) born in the same gestational week.

We had incomplete information on obesity (body mass index $\geq 30$ ) and therefore could not adjust for this potential confounder. To determine whether obesity might explain the observed associations for vascular dementia and Alzheimer's disease, we did sensitivity analyses assuming several obesity prevalences in women with a history of pre-eclampsia and a range of magnitudes for the association between obesity and dementia, using the array approach for testing the effect of an unmeasured or incompletely measured confounder. $^{21}$

\section{Patient involvement}

No patients were involved in setting the research question or the outcome measures, nor were they involved in the design or implementation of the study. No patients were asked to advise on interpretation or writing up of results. There are no plans to disseminate the results of the research to study participants.

\section{Results}

We followed 1178005 women, including 58410 with a history of pre-eclampsia, for 20352695 person years, with a median follow-up time of 21.1 (interquartile range 11.3-23.4) years per women. Table 1 shows the 
characteristics of the study cohort at the start of followup. The cohort consisted predominantly of younger women; $95.4 \%$ were under 45 years of age at the start of follow-up. Consequently, the median age at the end of follow-up was 49 years; only $10 \%$ of women were over 64 years of age at the end of follow-up. During the follow-up period, $1728(0.1 \%)$ women had a diagnosis of dementia registered (mean age at diagnosis 58.5 years); among these women, 105 (6.1\%) had an initial diagnosis of vascular dementia, 676 (39.1\%) were initially diagnosed as having Alzheimer's disease, and 947 (54.8\%) had a diagnosis of other/unspecified dementia.

Women with a history of pre-eclampsia had a 53\% increase in risk of dementia overall, compared with women with no history of pre-eclampsia (incidence rate for women with a history of pre-eclampsia: 11.6 per 100000 person years; incidence rate for women with no history of pre-eclampsia: 8.33 per 100000 person years; hazard ratio 1.53, 95\% confidence interval 1.26 to 1.85). Adjustment for preterm delivery or maternal age at first birth did not meaningfully change the magnitude of the association (adjustment for preterm delivery: hazard ratio $1.48,1.22$ to 1.79 ; adjustment for maternal age: $1.54,1.27$ to 1.87$)$. A history of pre-eclampsia seemed to be more strongly associated with late onset dementia (age $\geq 65$ years) than with early onset dementia (age $<65$ years) (P for age interaction $=0.05$; table 2 ). A history of recurrent pre-eclampsia was even more strongly associated with dementia than was a history of pre-eclampsia in a single pregnancy (pre-eclampsia in one pregnancy: hazard ratio $1.44,1.17$ to 1.76 ; pre-eclampsia in two or more pregnancies: $2.84,1.67$ to 4.81 ; $\mathrm{P}=0.02$ ). The amount of time elapsed since a woman's most recent delivery did not affect the strength of the association ( $<20$ years: hazard ratio $1.21,0.81$ to 1.81 ; $\geq 20$ years: $1.66,1.34$ to $2.06 ; \mathrm{P}=0.18)$. Magnitudes of association were similar for preterm pre-eclampsia (hazard ratio $1.58,0.95$ to 2.62 ) and term pre-eclampsia (1.54, 1.24 to 1.92) ( $\mathrm{P}$ for difference $=0.94$ ).

Women with a history of pre-eclampsia had a more than threefold increased risk of vascular dementia (incidence rate for women with a history of preeclampsia: 1.44 per 100000 person years; incidence rate for women with no history of pre-eclampsia: 0.47 per 100000 person years; fig 2 and table 2). In contrast, previous pre-eclampsia was associated with only a 45\% increase in the risk of Alzheimer's disease (incidence rate for women with a history of preeclampsia: 5.56 per 100000 person years; incidence rate for women with no history of pre-eclampsia: 3.28 per 100000 person years; $P$ for difference $=0.01$; fig 2 and table 2) and a 40\% increase in the risk of other/ unspecified dementia (incidence rate for women with a history of pre-eclampsia: 6.07 per 100000 person years; incidence rate for women with no history of pre-eclampsia: 4.58 per 100000 person years; fig 2 and table 2). Stratification by age suggested that a history of pre-eclampsia might be particularly strongly associated with the risk of late onset vascular dementia (hazard ratio $6.53,2.82$ to 15.1 ) (fig 2; table 2). We found little evidence of interaction between preeclampsia and age at onset of dementia for either Alzheimer's disease or other/unspecified dementia (fig 2; table 2).

Adjustment for cardiovascular disease, stroke, hypertension, chronic kidney disease, and diabetes developing during follow-up attenuated the results somewhat, particularly for vascular dementia. However, the associations remained strong and statistically significant (table 2; supplementary table A).

\section{Sensitivity analyses}

The results of sensitivity analyses on the sub-cohort from which we excluded women using antihypertensive

\begin{tabular}{|c|c|c|c|c|c|c|c|c|c|c|}
\hline & \multirow{2}{*}{$\begin{array}{l}\text { History of } \\
\text { pre-eclampsia }\end{array}$} & \multirow{2}{*}{$\begin{array}{l}\text { Person years } \\
\left(\times 10^{3}\right)\end{array}$} & \multicolumn{2}{|c|}{ Dementia overall } & \multicolumn{2}{|c|}{ Vascular dementia } & \multicolumn{2}{|c|}{ Alzheimer's disease } & \multicolumn{2}{|c|}{ Other/unspecified dementia } \\
\hline & & & No & $\mathrm{HR}(95 \% \mathrm{Cl})$ & No & HR $(95 \% \mathrm{Cl})$ & No & $\mathrm{HR}(95 \% \mathrm{Cl})$ & No & $\mathrm{HR}(95 \% \mathrm{Cl})$ \\
\hline \multicolumn{11}{|c|}{ Adjusted for potential confounders* } \\
\hline \multirow[t]{2}{*}{ Overall } & Yes & 972 & 113 & $1.53(1.26$ to 1.85$)$ & 14 & 3.46 (1.97 to 6.10$)$ & 40 & $1.45(1.05$ to 1.99$)$ & 59 & 1.40 (1.08 to 1.83$)$ \\
\hline & No & 19380 & 1615 & 1 (reference) & 91 & 1 (reference) & 636 & 1 (reference) & 888 & 1 (reference) \\
\hline \multicolumn{11}{|l|}{ Attained aget: } \\
\hline \multirow[t]{2}{*}{$<65$ years } & Yes & 957 & 73 & $1.35(1.06$ to 1.71$)$ & 7 & $2.32(1.06$ to 5.06$)$ & 19 & $1.12(0.70$ to 1.77$)$ & 47 & $1.37(1.02$ to 1.85$)$ \\
\hline & No & 18999 & 1152 & 1 (reference) & 65 & 1 (reference) & 382 & 1 (reference) & 705 & 1 (reference) \\
\hline \multirow[t]{2}{*}{$\geq 65$ years } & Yes & 15 & 40 & $2.02(1.46$ to 2.79$)$ & 7 & $6.53(2.82$ to 15.1$)$ & 21 & $1.96(1.26$ to 3.07$)$ & 12 & $1.51(0.84$ to 2.71$)$ \\
\hline & No & 381 & 26 & 1 (reference) & 26 & 1 (reference) & 254 & 1 (reference) & 183 & 1 (reference) \\
\hline \multicolumn{11}{|c|}{ Additionally adjusted for potential mediators $¥$} \\
\hline \multirow[t]{2}{*}{ Overall } & Yes & 972 & 113 & $1.26(1.03$ to 1.54$)$ & 14 & 2.21 (1.24 to 3.95$)$ & 40 & $1.38(1.00$ to 1.91$)$ & 59 & $1.11(0.85$ to 1.46$)$ \\
\hline & No & 19380 & 1615 & 1 (reference) & 91 & 1 (reference) & 636 & 1 (reference) & 888 & 1 (reference) \\
\hline \multicolumn{11}{|l|}{ Attained aget: } \\
\hline \multirow[t]{2}{*}{$<65$ years } & Yes & 957 & 73 & $1.10(0.87$ to 1.40$)$ & 7 & $1.41(0.64$ to 3.10$)$ & 19 & $1.08(0.68$ to 1.72$)$ & 47 & $1.13(0.84$ to 1.52$)$ \\
\hline & No & 18999 & 1152 & 1 (reference) & 65 & 1 (reference) & 382 & 1 (reference) & 705 & 1 (reference) \\
\hline \multirow[t]{2}{*}{$\geq 65$ years } & Yes & 15 & 40 & 1.65 (1.18 to 2.30$)$ & 7 & 4.17 (1.78 to 9.75$)$ & 21 & 1.85 (1.18 to 2.90$)$ & 12 & $1.08(0.60$ to 1.95$)$ \\
\hline & No & 381 & 26 & 1 (reference) & 26 & 1 (reference) & 254 & 1 (reference) & 183 & 1 (reference) \\
\hline
\end{tabular}




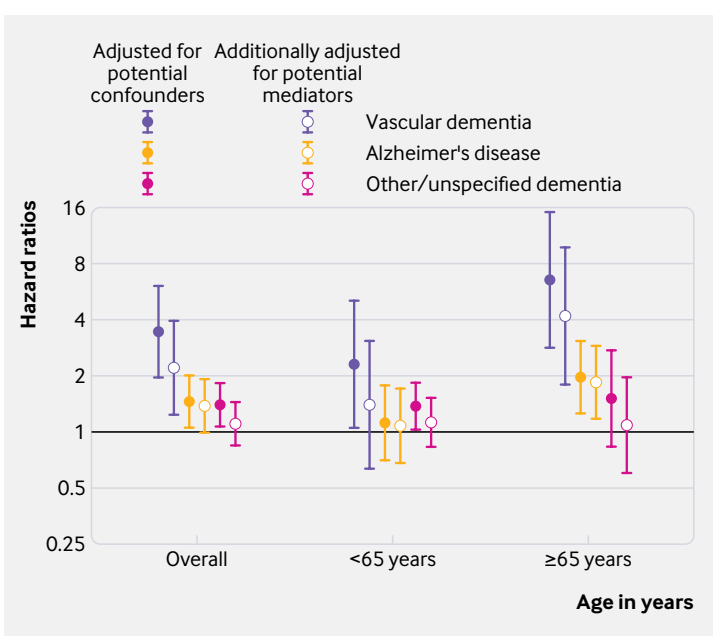

Fig 2 | Associations between history of pre-eclampsia and dementia, overall, by dementia subtype, and by attained age, in cohort of women with $\geq 1$ live birth or stillbirth in 1978-2015 in Denmark. Hazard ratios with $95 \% \mathrm{Cls}$ compare risks of vascular dementia, Alzheimer's disease, and other/unspecified dementia in women with history of pre-eclampsia and women with no history of pre-eclampsia, overall and separately for women $<65$ and $\geq 65$ years of age. All hazard ratios are adjusted for birth year ( 5 year intervals), parity $(1,2, \geq 3$ live births and/or stillbirths), and region of most recent delivery (potential confounders); age was underlying time scale in Cox model. Potential mediators additionally adjusted for were cardiovascular disease, stroke, hypertension, chronic kidney disease, and diabetes

drugs before the start of follow-up in 1995 did not differ from the results obtained in the full cohort (footnote to supplementary table A).

As dementia diagnoses are refined as the disease progresses, we did supplemental analyses based on the last dementia diagnosis a woman received during the study period. Although magnitudes of estimates for vascular dementia decreased somewhat, the strong association between pre-eclampsia and vascular dementia, particularly late onset vascular dementia, persisted (supplementary table B). Hazard ratios for Alzheimer's disease increased somewhat, showing a possible strong association between pre-eclampsia and late onset Alzheimer's disease, although the magnitudes of estimates for early and late onset Alzheimer's disease were not statistically significantly different $(\mathrm{P}=0.06$; supplementary table $\mathrm{B})$. Associations for other/unspecified dementia remained unchanged.

Analyses stratified by small for gestational age birth suggested that, compared with women with neither pre-eclampsia nor small for gestational age births, a history of pre-eclampsia with small for gestational age birth was associated with seven times the risk of vascular dementia (hazard ratio $7.33,2.66$ to 20.2), whereas pre-eclampsia without small for gestational age birth was associated with three times the risk $(3.51,1.80$ to 6.84$)$. We did not see similar differences for Alzheimer's disease (pre-eclampsia with small for gestational age birth: hazard ratio $0.86,0.36$ to 2.07 ; pre-eclampsia without small for gestational age birth: $1.59,1.18$ to 2.16 ) or other/unspecified dementia (pre-eclampsia with small for gestational age birth: 1.06, 0.40 to 2.83; pre-eclampsia without small for gestational age birth: $1.48,1.03$ to 2.13 ).

Sensitivity analyses suggested that body mass index was unlikely to be a strong enough confounder to explain the associations observed between preeclampsia and either dementia overall or vascular dementia (supplementary table C). Even if 30\% of women with a history of pre-eclampsia had a body mass index of 30.0 or higher and such a body mass index was associated with twice the risk of dementia observed in non-obese women (a stronger association than reported in the literature), the observed hazard ratio of 1.53 for overall dementia would have been biased by only $17.5 \%$, leading to an adjusted estimate of $1.30,1.07$ to 1.57 . Similarly, assuming a $30 \%$ prevalence of obesity among women with a history of pre-eclampsia and a relative risk of two or even three for the association between obesity and vascular dementia, the observed hazard ratio of 3.46 would have been biased by $16 \%$ to $29 \%$, leading to adjusted estimates of $2.98,1.70$ to 16.1 , and $2.68,1.53$ to 29.0 , respectively. In contrast, confounding by obesity might conceivably account for some or most of the observed association between pre-eclampsia and Alzheimer's disease (supplementary table C).

\section{Discussion}

In a cohort of more than a million women, a history of pre-eclampsia was associated with a modest (53\%) increase in the overall risk of dementia. Subtype specific associations were also modest for Alzheimer's disease and other/unspecified dementia but very strong for vascular dementia (threefold increase in risk) and late onset vascular dementia in particular (sixfold increase in risk).

\section{Strengths and limitations of study}

Our long study period and large cohort provided for long follow-up periods and allowed us to accrue a large number of outcomes, resulting in statistical power to examine the association between pre-eclampsia and subtypes of dementia, which has not previously been possible. Including all women in Denmark with pregnancies during the study period also minimised the possibility of selection bias. Moreover, use of prospectively collected register data eliminated the possibility of recall bias.

The study's potential limitations hinge on the validity and completeness of the register data on which the results are based. Although registration of pre-eclampsia diagnoses in the National Patient Register is incomplete (sensitivity 69\%), the validity of registered diagnoses is excellent (specificity $>99 \%$ ); the positive predictive value of a registered diagnosis is $74 \%$ overall and $100 \%$ for severe pre-eclampsia. ${ }^{22}$ The impact of misclassification of pre-eclampsia diagnoses on the observed associations is therefore likely to be negligible. Not all dementia is diagnosed, and 
registration of diagnosed dementia is probably also incomplete, as general practitioners, who often handle milder cases of dementia, do not report to the National Patient Register. ${ }^{23}$ However, a study of dementia diagnoses registered in Danish hospital registers in 2003 found that $88 \%$ of people with a registered diagnosis of dementia did in fact have dementia according to their medical records. ${ }^{24}$ Registered diagnoses of Alzheimer's disease, vascular dementia, and other/unspecified dementia agreed with the diagnosis noted in the medical record for $97 \%$, $96 \%$, and $81 \%$ of patients, respectively. ${ }^{24}$ Furthermore, whether dementia in our study population was diagnosed or registered would not have depended on history of pre-eclampsia. Incomplete registration of mild dementia might have affected the generalisability of our results, but only if the association with preeclampsia differs by severity of dementia.

Almost $55 \%$ of the dementia in our cohort was registered as other/unspecified dementia, reflecting both the clinical challenges involved in diagnosing and subtyping dementia and the fact that a substantial proportion of patients have "mixed" forms of dementia, making them difficult to classify. ${ }^{23}$ The observed hazard ratios suggest that our other/unspecified dementia group did not have a preponderance of characteristics of vascular dementia, as effect magnitudes more closely resembled those estimated for Alzheimer's disease than for vascular dementia.

As might be expected for a pregnancy cohort, but unusually for a cohort being followed for dementia, the women in our study were still relatively young at the end of follow-up ( $90 \%$ were $<65$ years of age). Consequently, only $0.1 \%$ of women were diagnosed as having dementia during follow-up, and the rates of dementia observed were much lower than one would expect had the study included older women. ${ }^{25}$ However, the observed rates of dementia compare favourably with incidences reported in studies of early onset dementia (incidence among people 45-64 years of age, 2.4-11.9 per $100000^{26}$ ). Our cohort was also very ethnically homogeneous, being comprised predominantly of women of Scandinavian descent, which might limit its generalisability to other populations.

We did not have complete information on smoking, socioeconomic status, and body mass index, so we could not directly evaluate their impact on our results. However, as smoking is negatively associated with preeclampsia, confounding by smoking cannot explain the observed associations between pre-eclampsia and dementia. Moreover, although the association between education and dementia is well known, confounding by educational level and associated socioeconomic status is most likely small given Denmark's free, universal healthcare and educational systems. Sensitivity analyses suggested that confounding by obesity was unlikely to explain the observed associations for vascular dementia; in contrast, obesity could conceivably explain a considerable part of the association between pre-eclampsia and Alzheimer's disease. Beyond these three variables of particular interest, we also cannot rule out the possibility of residual confounding by other unmeasured covariates.

\section{Possible explanations for findings}

Pregnancy is often characterised as a stress test with implications for a woman's future risk of cardiovascular and microvascular disease. Pre-eclampsia (a "failed" stress test) is associated with a range of later vascular conditions, ${ }^{4-7}$ making links with dementia plausible as well. Two previous epidemiological studies found support for a link between preeclampsia and Alzheimer's disease, ${ }^{14} 27$ and a third suggested the possibility of an association with vascular dementia. ${ }^{13}$ Other epidemiological studies found little evidence of an association, ${ }^{12}$ possibly owing to a lack of statistical power. However, a link between pre-eclampsia and dementia is biologically plausible. Vascular (endothelial) dysfunction and heightened inflammatory responses are implicated in the pathophysiology of pre-eclampsia, ${ }^{27}$ and they are also thought to play a role in the pathophysiology of dementia, particularly vascular dementia, ${ }^{28}$ with large vessel atherosclerosis, small vessel arteriosclerosis, and angiopathy eventually leading to ischaemic lesions, cerebrovascular infarcts, and cognitive decline. Neuroimaging studies have shown that women with previous pre-eclampsia have more white matter lesions than do women whose pregnancies were normotensive ${ }^{10}$; white matter lesions have been linked with cognitive decline and dementia. ${ }^{29} 30$ Furthermore, a history of pre-eclampsia has recently been shown to be associated with an increased risk of cognitive impairment, with previously pre-eclamptic women showing cognitive changes typical of those observed in vascular dementia. ${ }^{31}$ Genetic expression studies have also led to speculation that the so-called pre-eclampsia susceptibility gene STOX1, which is expressed in both placenta and brain, might play a role in Alzheimer's disease. In particular, van Dijk and colleagues showed that a specific STOX1 isoform was overexpressed in late onset Alzheimer's disease and controlled a pathway involved in the processing of amyloid- $\beta$ protein precursor. ${ }^{11}$ Our finding that pre-eclampsia was strongly associated with vascular dementia adds convincing epidemiological evidence to suggest that the two conditions may share underlying mechanisms or susceptibility pathways. In contrast, we found little evidence to support an association between pre-eclampsia and Alzheimer's disease, particularly after accounting for the potential effect of obesity.

Hypertension, cardiovascular disease, stroke, and diabetes are shared risk factors for both pre-eclampsia and dementia. Adjustment for these common risk factors decreased the magnitude of the estimates somewhat, indicating that some of the associations between pre-eclampsia and dementia may be mediated by vascular risk factors and comorbidities. Nevertheless, meaningful-and strong, in the case of vascular dementia-associations persisted after 
adjustment for these comorbidities, suggesting that the pathway between pre-eclampsia and dementia may also involve additional mechanisms beyond those linking pre-eclampsia, cardiovascular disease, stroke, hypertension, and diabetes.

\section{Conclusions and implications}

As lifespans worldwide continue to increase, cases of dementia are expected to triple by $2050,{ }^{32}$ adding to the burgeoning costs of caring for ageing populations. Countering this development will require strategies for early identification of people at risk of dementia and interventions to delay or prevent the onset of disease. Our findings suggest that asking about a history of preeclampsia could help physicians to identify a group of women who might benefit from early attention to modifiable vascular risk factors for dementia, such as concerted efforts to reduce blood pressure and blood cholesterol concentrations. Our findings also support a common underlying mechanism for pre-eclampsia and vascular dementia, suggesting an avenue for future studies of candidate biological pathways and targets for intervention.

Contributors: SB conceived and designed the study, acquired and analysed the data, interpreted the study findings, and drafted the manuscript. JW designed the study, advised on selection and implementation of analytical methods, interpreted the study findings, and critically reviewed the manuscript. HAB designed the study, supervised and directed the conduct of the study, interpreted the study findings, and critically revised the manuscript. All authors had full access to all of the data in the study and can take responsibility for the integrity of the data and the accuracy of the data analysis. The corresponding author attests that all listed authors meet authorship criteria and that no others meeting the criteria have been omitted. $\mathrm{HAB}$ is the guarantor.

Funding: This work was supported by the Danish Council for Independent Research (DFF-4092-00213). The Danish Council for Independent Research had no role in the design and conduct of the study; collection, management, analysis, and interpretation of the data; preparation, review, or approval of the manuscript; or the decision to submit the manuscript for publication. The researchers acted independently from the study sponsor in all aspects of this study. Competing interests: All authors have completed the ICMJE uniform disclosure form at www.icmje.org/coi_disclosure.pdf (available on request from the corresponding author) and declare: SB was supported by a grant from the Danish Council for Independent Research; no financial relationships with any organisations that might have an interest in the submitted work in the previous three years; no other relationships or activities that could appear to have influenced the submitted work.

Ethical approval: Studies based solely on data from the Danish national registers do not need approval from the Danish research bioethics committees, as study participants are never contacted, and consent is not required for the use of register information. The study's use of register data was covered by the approval extended by the Danish Data Protection Agency to all register based studies conducted by Statens Serum Institut (approval No 2015-57-0102).

Data sharing: This study is based on Danish national register data. These data do not belong to the authors but to the Danish Ministry of Health, and the authors are not permitted to share them, except in aggregate (as, for example, in a publication). However, interested parties can obtain the data on which the study was based by submitting a research protocol to the Danish Data Protection Agency (Datatilsynet) and then, once permission has been received, applying to the Ministry of Health's Research Service (Forskerservice) at forskerservice@ssi.dk.

Transparency: The guarantor affirms that the manuscript is an honest, accurate, and transparent account of the study being reported; that no important aspects of the study have been omitted; and that any discrepancies from the study as planned (and, if relevant, registered) have been explained.
This is an Open Access article distributed in accordance with the Creative Commons Attribution Non Commercial (CC BY-NC 4.0) license, which permits others to distribute, remix, adapt, build upon this work non-commercially, and license their derivative works on different terms, provided the original work is properly cited and the use is noncommercial. See: http://creativecommons.org/licenses/by-nc/4.0/.

1 ACOG Committee on Obstetric Practice, American College of Obstetricians and Gynecologists. ACOG practice bulletin. Diagnosis and management of preeclampsia and eclampsia. Number 33, January 2002. Int J Gynaecol Obstet 2002;77:67-75.

2 Powe CE, Levine RJ, Karumanchi SA. Preeclampsia, a disease of the maternal endothelium: the role of antiangiogenic factors and implications for later cardiovascular disease. Circulation 2011;123:2856-69. doi:10.1161/CIRCULATIONAHA.109.853127

3 Brennan LJ, Morton JS, Davidge ST. Vascular dysfunction in preeclampsia. Microcirculation 2014:21:4-14. doi:10.1111/micc.12079

4 Behrens I, Basit S, Melbye M, et al. Risk of post-pregnancy hypertension in women with a history of hypertensive disorders of pregnancy: nationwide cohort study. BMJ 2017;358:j3078. doi:10.1136/bmj.j3078

5 Wu P, Haththotuwa R, Kwok CS, et al. Preeclampsia and future cardiovascular health: a systematic review and meta-analysis. Circ Cardiovasc Qual Outcomes 2017;10:e003497. doi:10.1161/CIRCOUTCOMES.116.003497

6 Wu P, Kwok CS, Haththotuwa R, et al. Pre-eclampsia is associated with a twofold increase in diabetes: a systematic review and meta-analysis. Diabetologia 2016;59:2518-26. doi:10.1007/s00125-016-4098-x

7 Brown MC, Best KE, Pearce MS, Waugh J, Robson SC, Bell R. Cardiovascular disease risk in women with pre-eclampsia: systematic review and meta-analysis. Eur J Epidemiol 2013;28:1-19. doi:10.1007/s10654-013-9762-6

8 Postma IR, Bouma A, Ankersmit IF, Zeeman GG. Neurocognitive functioning following preeclampsia and eclampsia: a longterm follow-up study. Am J Obstet Gynecol 2014;211:37.e1-9. doi:10.1016/j.ajog.2014.01.042

9 Wiegman MJ, Zeeman GG, Aukes AM, et al. Regional distribution of cerebral white matter lesions years after preeclampsia and eclampsia. Obstet Gynecol 2014;123:790-5. doi:10.1097/ AOG.0000000000000162

10 Mielke MM, Milic NM, Weissgerber TL, et al. Impaired cognition and brain atrophy decades after hypertensive pregnancy disorders. Circ Cardiovasc Qual Outcomes 2016;9(Suppl 1):S70-6. doi:10.1161/CIRCOUTCOMES.115.002461

11 van Dijk M, van Bezu J, Poutsma A, et al. The pre-eclampsia gene STOX1 controls a conserved pathway in placenta and brain upregulated in late-onset Alzheimer's disease. J Alzheimers Dis 2010;19:673-9. doi:10.3233/JAD-2010-1265

12 Nelander M, Cnattingius S, Åkerud H, Wikström J, Pedersen NL, Wikström AK. Pregnancy hypertensive disease and risk of dementia and cardiovascular disease in women aged 65 years or older: a cohort study. BMJ Open 2016;6:e009880. doi:10.1136/bmjopen-2015-009880

13 Andolf EG, Sydsjö GCM, Bladh MK, Berg G, Sharma S. Hypertensive disorders in pregnancy and later dementia: a Swedish National Register Study. Acta Obstet Gynecol Scand 2017;96:464-71. doi:10.1111/aogs.13096

14 Abheiden CNH, van Doornik R, Aukes AM, van der Flier WM, Scheltens P, de Groot C). Hypertensive disorders of pregnancy appea not to be associated with Alzheimer's disease later in life. Dement Geriatr Cogn Dis Extra 2015;5:375-85. doi:10.1159/000439043

15 Schmidt M, Pedersen L, Sørensen HT. The Danish Civil Registration System as a tool in epidemiology. Eur J Epidemiol 2014;29:541-9. doi:10.1007/s10654-014-9930-3

16 Knudsen LB, Olsen J. The Danish Medical Birth Registry. Dan Med Bull 1998;45:320-3.

17 Lynge E, Sandegaard JL, Rebolj M. The Danish National Patient Register. Scand J Public Health 2011;39(Suppl):30-3. doi:10.1177/1403494811401482

18 Helweg-Larsen K. The Danish Register of Causes of Death. Scand I Public Health 2011;39(Suppl):26-9. doi:10.1177/1403494811399958

19 Lau B, Cole SR, Gange SJ. Competing risk regression models for epidemiologic data. Am J Epidemiol 2009;170:244-56. doi:10.1093/aje/kwp107

20 Lin DY, Wei LJ, Ying Z. Checking the Cox model with cumulative sums of martingale-based residuals. Biometrika 1993;80:557-72 doi:10.1093/biomet/80.3.557.

21 Schneeweiss S. Sensitivity analysis and external adjustment for unmeasured confounders in epidemiologic database studies of therapeutics. Pharmacoepidemiol Drug Saf 2006;15:291-303. doi:10.1002/pds.1200 
22 Klemmensen ÅK, Olsen SF, Østerdal ML, Tabor A. Validity of preeclampsia-related diagnoses recorded in a national hospital registry and in a postpartum interview of the women. $\mathrm{Am}$ J Epidemiol 2007;166:117-24. doi:10.1093/aje/kwm139

23 Phung TKT, Waltoft BL, Kessing LV, Mortensen PB, Waldemar G. Time trend in diagnosing dementia in secondary care. Dement Geriatr Cogn Disord 2010;29:146-53. doi:10.1159/000269933

24 Phung TKT, Andersen BB, Høgh P, Kessing LV, Mortensen PB, Waldemar G. Validity of dementia diagnoses in the Danish hospital registers. Dement Geriatr Cogn Disord 2007;24:220-8. doi:10.1159/000107084

25 Fiest KM, Jetté N, Roberts II, et al. The prevalence and incidence of dementia: a systematic review and meta-analysis. Can I Neurol Sci 2016;43(Suppl 1):S3-50. doi:10.1017/cjn.2016.18

26 Vieira RT, Caixeta L, Machado S, et al. Epidemiology of early-onset dementia: a review of the literature. Clin Pract Epidemiol Ment Health 2013;9:88-95. doi:10.2174/1745017901309010088

27 Amaral LM, Cunningham MWJr, Cornelius DC, LaMarca B.

Preeclampsia: long-term consequences for vascular health. Vasc Health Risk Manag 2015;11:403-15.
28 O'Brien JT, Thomas A. Vascular dementia. Lancet 2015;386:1698-706. doi:10.1016/S0140-6736(15)00463-8

29 Prins ND, van Dijk EJ, den Heijer T, et al. Cerebral white matter lesions and the risk of dementia. Arch Neurol 2004;61:1531-4. doi:10.1001/archneur.61.10.1531

30 Debette S, Markus HS. The clinical importance of white matter hyperintensities on brain magnetic resonance maging: systematic review and meta-analysis. BMJ 2010;341:c3666. doi:10.1136/bmj.c3666

31 Fields JA, Garovic VD, Mielke MM, et al. Preeclampsia and cognitive impairment later in life. Am / Obstet Gynecol 2017;217:74.e1-11. doi:10.1016/j.ajog.2017.03.008

32 Wortmann M. Dementia: a global health priority - highlights from an ADI and World Health Organization report. Alzheimers Res Ther 2012;4:40.

\section{Supplementary tables}

